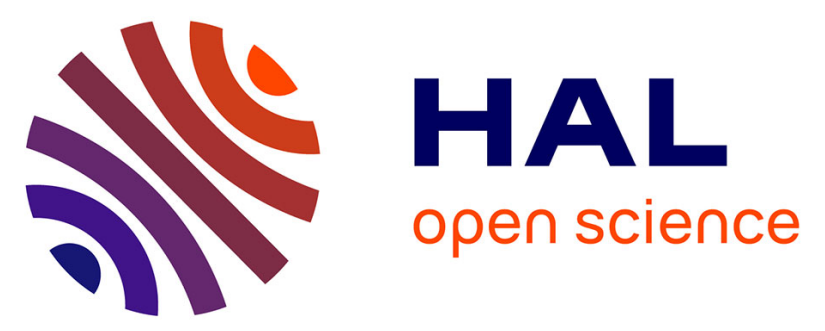

\title{
Revealing the Interplay between Adsorbed Molecular Layers and Gold Nanoparticles by Linear and Nonlinear Optical Properties
}

Laetitia Dalstein, Maroua Ben Haddada, Grégory Barbillon, Christophe Humbert, Abderrahmane Tadjeddine, Souhir Boujday, Bertrand Busson

\section{To cite this version:}

Laetitia Dalstein, Maroua Ben Haddada, Grégory Barbillon, Christophe Humbert, Abderrahmane Tadjeddine, et al.. Revealing the Interplay between Adsorbed Molecular Layers and Gold Nanoparticles by Linear and Nonlinear Optical Properties. Journal of Physical Chemistry C, 2015, 119 (30), pp.17146-17155. 10.1021/acs.jpcc.5b03601 . hal-02111041

\author{
HAL Id: hal-02111041 \\ https://hal.science/hal-02111041
}

Submitted on 14 May 2019

HAL is a multi-disciplinary open access archive for the deposit and dissemination of scientific research documents, whether they are published or not. The documents may come from teaching and research institutions in France or abroad, or from public or private research centers.
L'archive ouverte pluridisciplinaire HAL, est destinée au dépôt et à la diffusion de documents scientifiques de niveau recherche, publiés ou non, émanant des établissements d'enseignement et de recherche français ou étrangers, des laboratoires publics ou privés. 


\title{
Revealing the Interplay Between Adsorbed Molecular
}

\section{Layers and Gold Nanoparticles by Linear and}

\section{Non-Linear Optical Properties}

Laetitia Dalstein, ${ }^{\dagger}$ Maroua Ben Haddada, ${ }^{\ddagger}, \mathbb{I}$ Grégory Barbillon, ${ }^{\S}$ Christophe

Humbert, ${ }^{\dagger}$ Abderrahmane Tadjeddine ${ }^{\dagger}$ Souhir Boujday, ${ }^{\ddagger}, \mathbb{I}$ and Bertrand Busson*, $\uparrow$

Univ. Paris-Sud, Laboratoire de Chimie Physique, CNRS, Bâtiment 201 Porte 2, 91405 Orsay, France, Sorbonne Universités, UPMC Univ. Paris 6, UMR CNRS 7197, Laboratoire de Réactivité de Surface, F75005 Paris, France, CNRS, UMR 7197, Laboratoire de Réactivité de Surface, F75005 Paris, France, and Univ. Paris-Sud, Institut d'Electronique Fondamentale, CNRS UMR 8622, Bâtiment 220, 91405 Orsay, France

E-mail: bertrand.busson@u-psud.fr

\begin{abstract}
Gold nanoparticles (AuNPs) chemically grafted on substrates are widely used as sensors due to their plasmonic properties. The efficiency and robustness of such sensors strongly depend on the molecular sublayer structure, which influences the distribution of AuNPs, and therefore the plasmonic properties of the layer. Few spectroscopic tools are able to sense the

*To whom correspondence should be addressed

$\dagger$ Univ. Paris-Sud, Laboratoire de Chimie Physique, CNRS, Bâtiment 201 Porte 2, 91405 Orsay, France

†Sorbonne Universités, UPMC Univ. Paris 6, UMR CNRS 7197, Laboratoire de Réactivité de Surface, F75005

ITCNRS, UMR 7197, Laboratoire de Réactivité de Surface, F75005 Paris, France

${ }^{\S}$ Univ. Paris-Sud, Institut d'Electronique Fondamentale, CNRS UMR 8622, Bâtiment 220, 91405 Orsay, France
\end{abstract} Paris, France 
grafting layer both before and after particle deposition. Here, we use Sum-Frequency Generation (SFG) spectroscopy to deeply investigate both the grafting layer and the immobilized AuNPs. We combine SFG to reflectance UV-Visible spectroscopy and Scanning Electron Microscopy (SEM) for $14 \mathrm{~nm}$ diameter AuNPs, dispersed on modified silicon surfaces with either amine or mixed amine/thiol terminated layers. SFG spectra show the specific vibrational fingerprint of each supporting layer through the amplitudes of methylene and methyl vibration modes, and prove the presence of unreacted ethoxy groups from APTES. We establish a linear evolution of the absorbance amplitudes with AuNP surface coverage, a relationship valid up to the aggregation limit of $10^{11}$ AuNPs. $\mathrm{cm}^{-2}$. In the same way, SFG amplitudes follow a quadratic dependence with the UV-Vis absorbance amplitudes, showing the close correlation between non-linear and linear optical properties. In addition, the optical properties of the AuNP layers are stable for several months (plasmon position and damping) despite their storage in ambient air and long exposure to visible laser light.

\section{Introduction}

Over the last several decades, the use of materials with plasmonic properties has been expanding rapidly. ${ }^{1-4}$ Among the various uses of plasmonics, surface sensing has greatly benefitted from the optical enhancements due to the interaction of light with surface plasmons. ${ }^{1}$ The use of localized surface plasmon resonance, surface roughness or metallic nanoparticles typically leads to dramatic improvements in the performance and sensitivity of devices. Using nano-objects on a substrate offers the advantage of a tailor-made production, with a large choice of sizes, shapes, and chemical composition, depending on the properties sought. Gold nanoparticles (AuNP) are routinely used because they associate a high chemical stability with plasmonic properties tunable in the visible and in the near-infrared ranges. Their asset lies in the localized surface plasmon resonance (LSPR) of the individual nano-objects. LSPR strongly depends on the shape, size, environment, and chemical composition of the nanoparticles. Gold nanospheres with 10-20 $\mathrm{nm}$ diameter are a textbook example, with one SPR in the green region of the spectrum, around $525 \mathrm{~nm}$ in aqueous 
solution. ${ }^{2,5-7}$ Plasmonic properties are also modified upon interaction at the surface of a substrate. Controlling the distance between particles (average and distribution) grafted on a surface remains one of the challenges of such a synthesis of complex materials. ${ }^{8-11}$

Metallic nanoparticles are usually grafted on surfaces through wet chemistry procedures where NPs are first synthesized in solution, then immobilized on prefunctionalized surfaces. On gold substrates, grafting is achieved through a self-assembled monolayer (SAM) of thiols, whereas silanes are chosen for silicon or silica-like materials, with a large choice of available terminal functional groups, providing a simple approach for adsorption of the nanoparticles with an amine group, ${ }^{12-14}$ as well as more elaborate chemical transformations (e.g. gold grafting through a thiol termination $^{8-10}$ or bidendate ligands like lipoid acid ${ }^{15}$ ). The quality of a substrate/SAM/AuNP assembly may be defined in terms of surface homogeneity (or designed heterogeneity ${ }^{16}$ ), structural quality, and thickness of the silane layer, surface density and mean distance between the grafted particles, and plasmonic properties of the individual or aggregated particles. Reproducibility and simplicity of the chemical preparation also condition the large-scale use of the overall method. This makes it crucial to be able to control the quality of substrate/SAM/AuNP systems.

UV-Visible spectroscopy, in transmission or reflection mode, is a reliable tool for investigating the absorption properties of AuNP layers. Coupling to atomic force or electron microscopy makes it possible to correlate plasmonic properties to the distribution of particles on the surface. ${ }^{12-17} \mathrm{X}$ Ray Photoelectron spectroscopy (XPS) provides valuable data on SAMs, however it does not support in situ analysis during self-assembly and grafting at the solid-liquid interface. The chemical and structural analysis of SAMs greatly benefits from enhanced vibrational spectroscopies. Surface enhanced Raman scattering (SERS) sensitivity can reach the single molecule level ${ }^{18}$ but can only be used to characterize the layer after deposition of gold nanoparticles. Surface infrared methods like IRRAS (Infrared reflection absorption spectroscopy), ${ }^{19}$ ATR (Attenuated Total Reflectance), ${ }^{20}$ or multiple-reflection infrared spectroscopy ${ }^{21}$ allow for the characterization of a monolayer on sil- 
icon but the addition of the gold objects add significant complications to the analysis because of the presence of surfactants, and most of these techniques lack coupling to the plasmonic properties of the AuNPs. Sum-frequency generation spectroscopy (SFG) is sensitive enough to study the bare monolayer, and benefits from an enhancement due to the SPR when gold nanospheres are present. ${ }^{22}$ In addition, it should be sensitive to the structures located under the gold layer.

Several kinds of SAMS have been studied by SFG, mostly thiols ${ }^{22-25}$ and silanes. Studies on silanes mostly concentrate on the adsorption on silicon and quartz, and monitor layer growth and order through the $\mathrm{CH}$ stretches, in particular the terminal $\mathrm{CH}_{3}$ groups from alkyl silanes. ${ }^{26-32}$ Aminoalkyl silanes have the additional advantage over alkylsilanes that they are provided with a free amine function, which can be used to attach AuNPs through electrostatic interaction, as mentioned above. Such films have also been investigated with SFG in pure ${ }^{33}$ or mixed films. ${ }^{34}$

In this work, we investigate, at each step, the chemical and optical properties of several types of $\mathrm{Si} / \mathrm{SAM} / \mathrm{AuNP}$ systems. The different chemical functions of the SAMs, amine and/or acid, led to various densities and aggregation states of the deposited particles, measured by scanning electron microscopy and UV-visible reflectance spectroscopy. SFG is used to investigate the structure of the SAM and the properties of the AuNP layers. We correlate the linear and non-linear optical properties of the AuNP layers and discuss them with respect to the local density of particles, whether aggregated or not, on silica surfaces.

\section{Experimental details}

\section{Materials}

N-ethyl-N'-(3-(dimethylamino)propyl) carbodiimide hydro-chloride (EDC, 98\%), sodium citrate ( $\left.\mathrm{HOC}(\mathrm{COONa})\left(\mathrm{CH}_{2} \mathrm{COONa}\right)_{2} \cdot 2 \mathrm{H}_{2} \mathrm{O}, 99 \%\right)$, gold(III)chloride trihydrate $\left(\mathrm{HAuCl}_{4}, 3 \mathrm{H}_{2} \mathrm{O}, 99.9 \%\right)$, tannic acid $\left(\mathrm{C}_{76} \mathrm{H}_{52} \mathrm{O}_{46}, 99 \%\right)$, dodecanethiol $\left(\mathrm{CH}_{3}\left(\mathrm{CH}_{2}\right)_{11} \mathrm{SH}, 98 \%\right)$ and thiophenol $\left(\mathrm{C}_{6} \mathrm{H}_{5} \mathrm{SH}\right.$, 
99\%) were purchased from Sigma Aldrich. N-hydroxysuccinimide (NHS, 97\%), 11-mercaptoundecanoic acid (MUA, 95\%) and (3-aminopropyl) triethoxysilane (APTES, 99\%) were purchased from Aldrich. N-doped Silicon wafer $<100>$ from Siltronix was cut into $2 \times 2 \mathrm{~cm}^{2}$ pieces. Sulfuric acid $\left(\mathrm{H}_{2} \mathrm{SO}_{4}\right)$, $96 \%$ and hydrogen peroxide $\left(\mathrm{H}_{2} \mathrm{O}_{2}\right), 30 \%$ in water were supplied by Carlo Erba. Toluene, acetone, and ethanol were purchased from Analar Normapur. MilliQ water (18 MS.cm, Millipore, France) was used for the preparation of aqueous solutions and for all rinses. All chemicals were reagent grade or higher and were used without further purification.

\section{Surface Functionalization}

First, silicon surfaces were cleaned following a procedure which includes several washing steps, a piranha solution treatment, and finally UV-ozone cleaning. A detailed description of this procedure is given in reference. ${ }^{20}$ The same experimental conditions were applied for silane grafting for the amine-terminated silane (APTES): oxidized and cleaned silicon wafers were immersed in a 50 $m M$ solution of silane in anhydrous toluene at $75^{\circ} \mathrm{C}$ for $24 h$. After silanization, the samples were washed twice, sonicated for $10 \mathrm{~min}$ in anhydrous toluene, dried under nitrogen, and heated at $90^{\circ} \mathrm{C}$ for $2 h$. The MUA grafting was achieved after APTES deposition on silicon surfaces. First, MUA was activated in solution using a mixture of EDC and NHS in ethanol for $90 \mathrm{~min}$. Then the APTES-modified silicon surfaces were placed in the activated solution of MUA for $90 \mathrm{~min}$, then washed twice in ethanol and dried under nitrogen (Figure 1(a)). The corresponding interfaces will be referred to as Si/SAM.

\section{Gold nanoparticles preparation and deposition}

Colloidal gold particles were prepared according to the tannic acid method of Slot and Geuze. ${ }^{35}$ To produce $100 \mathrm{~mL}$ of a $14 \mathrm{~nm}$ diameter colloid, two stock solutions were prepared: solution A: $1 \mathrm{~mL}$ $1 \%$ (w/v) $\mathrm{HAuCl}_{4}$ and $79 \mathrm{~mL}$ deionized water; solution B: $4 \mathrm{~mL} 1 \%$ sodium citrate, $0.025 \mathrm{~mL} 1 \%$ tannic acid and $16 \mathrm{~mL}$ deionized water. Solutions A and B were heated to $60^{\circ} \mathrm{C}$ and mixed while stirring. Once solution turned red, the mixture was heated up to $95^{\circ} \mathrm{C}$ and cooled on ice. Gold 
nanoparticle size was confirmed by transmission electron microscopy indicating an average size of $13.5 \mathrm{~nm}$, and the concentration of AuNP in the solution is $3.34 \mathrm{nmol. \textrm {L } ^ { - 1 }}$. Freshly synthesized AuNPs were then deposited, with no further dilution, on the Si/SAM surfaces for 30 or 60 min in a sonication bath (Elma, $90 \mathrm{~W}, 45 \mathrm{kHz}$ ). Finally, silicon substrates were washed twice in water and dried under nitrogen. The corresponding interfaces will be referred to as $\mathrm{Si} / \mathrm{SAM} / \mathrm{AuNP}$ in the following. Some samples were functionalized with dodecanethiol (DDT) or thiophenol (TP) by dipping them in an ethanol solution at $10 \mathrm{mM}$ for $12 \mathrm{~h}$. The corresponding interfaces are referred to as $\mathrm{Si} / \mathrm{SAM} / \mathrm{AuNP} / \mathrm{DDT}$ (resp. Si/SAM/AuNP/TP) in the following.

\section{Scanning electron microscopy}

Images of the gold nanoparticles on the modified silicon wafers were obtained using a SEM FEG Hitachi SU-8000 scanning electron microscope (SEM) with a voltage of $15 \mathrm{kV}$ and a working

distance of 5.9-10.1 $\mathrm{mm}$. Images were analyzed using the free software Image ${ }^{36}$ to measure the surface density of nanoparticles. We determine the number of nanoparticles by counting them individually for three different images at least in three different regions of the samples. As it is not possible to determine the exact number of particles present in clusters, we did not consider clusters and established estimated values. SEM images were all recorded after the DDT (resp. TP) functionalization step.

\section{UV-Visible spectroscopy}

Silicon substrates are not transparent in the probed optical range $(400-800 \mathrm{~nm})$, thus transmission mode could not be used. UV-Visible absorbance spectra were recorded in specular reflection geometry with a Cary-5000 spectrophotometer Agilent at an incidence angle of $55^{\circ}$. We used a UV-Vis VeeMAX accessory from PIKE Technologies. Polarization was adjusted parallel ( $p$ ) or perpendicular $(s)$ to the plane of incidence, $(x, z)$ in Figure 1 (more details in the supplementary information). A reference spectrum of the bare silicon substrate was first recorded to obtain the optical signature of the grafted AuNps by differential measurement. In these conditions, the mea- 
surement reproducibility is ensured at a level greater than $10^{-3}$ absorbance units.

\section{Non-linear optical vibrational spectroscopy}

Sum-Frequency Generation spectra were recorded by using a setup similar to the one described in reference. ${ }^{17}$ It is based on a 6.8 ps pulsed laser $\mathrm{Nd}: \mathrm{YVO}_{4}$ source coupled to an Acousto Optic Modulator (62.5 $\mathrm{MHz}$ micropulse repetition rate, $2 \mu \mathrm{s}$ train, $25 \mathrm{~Hz}$ macropulse repetition rate). After amplification, one part of the beam is used to pump an infrared optical parametric oscillator (OPO) built around a $\mathrm{LiNBO}_{3}$ crystal giving access to the $2500-4000 \mathrm{~cm}^{-1}$ spectral range $(10 \mu \mathrm{J}$ pulse energy, $3 \mathrm{~cm}^{-1}$ OPO bandwidth). The other part is used to obtain a tunable visible beam (5 $\mu J$ pulse energy, 440-710 $\mathrm{nm}$ wavelength) by frequency conversion in an OPO built around a BBO crystal. The infrared and visible beams are then mixed in a co-propagating geometry at the same point of the probed surface for each sample. The incidence angles are therefore $65^{\circ}$ and $55^{\circ}$ with respect to the surface normal, respectively. The infrared, visible, and SFG beams are p-polarized. All the SFG data are recorded in the same experimental conditions as depicted in Figure 1(b) with the same infrared (IR) and visible powers. A reference measurement on a $\mathrm{ZnS}$ crystal is performed simultaneously to take into account possible intensity fluctuations. For a fixed visible wavelength, the SFG spectra are recorded as a function of the IR wavelength in the 3.0-3.6 $\mu \mathrm{m}$ spectral range of the $\mathrm{CH}$ stretch to probe the alkyl vibration of the APTES and APTES/MUA supporting layers. In these conditions, the SFG photons are detected by photomultipliers after spatial and spectral filtering through a monochromator.

The SFG intensity is directly proportional to the sample response: ${ }^{37,38} \mathrm{I}_{S F G} \propto\left|\chi_{e f f}^{(2)}\right|^{2} \mathrm{I}_{V I S} \mathrm{I}_{I R}$ with $\mathrm{I}_{V I S}, \mathrm{I}_{I R}$ the intensities of the visible and IR incident beams, respectively. $\chi_{e f f}^{(2)}$ is the effective non-linear second order susceptibility of the interface, which can be written as $\chi_{e f f}^{(2)}=\chi_{S i / A u}^{(2)}+$ $\chi_{m}^{(2)} \cdot \chi_{S i / A u}^{(2)}$ is the second-order non-linear susceptibility of silicon and gold nanoparticles (when the latter are present, and $\chi_{S i / A u}^{(2)}$ is essentially constant in a vibrational spectrum, hence often called the non-resonant contribution to the SFG signal). $\chi_{m}^{(2)}$ is the second-order non-linear susceptibility of the organic layers. The SFG signal is therefore modelled by the complex second order suscep- 


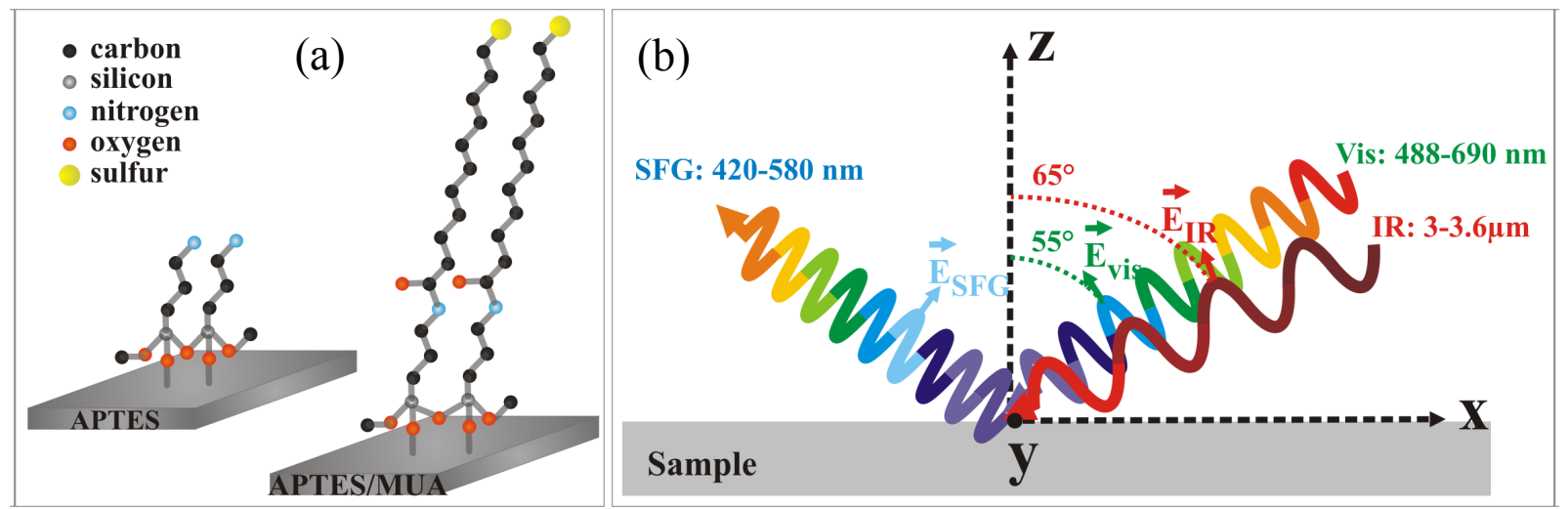

Figure 1: (a) Schematic of the expected silane layers; (b) SFG experimental configuration: $\mathrm{XZ}$ is the incidence plane of the SFG, Vis, IR beams in a $p p p$ polarization scheme.

tibilities of gold nanoparticles $\left(\chi_{S i / A u}^{(2)}\right)$ and the organic molecular layer $\left(\chi_{m}^{(2)}\right)$ by analogy with a metal-molecule interface: ${ }^{39} \mathrm{I}_{S F G} \propto\left|\chi_{S i / A u}^{(2)}+\chi_{m}^{(2)}\right|^{2}$ where $\chi_{S i / A u}^{(2)}$ and $\chi_{m}^{(2)} 40$ are complex values, and interfere constructively or destructively depending on the modulus and phase of $\chi_{S i / A u}^{(2)} \cdot{ }^{41,42}$

\section{Results and discussion}

\section{Characterization of silane layers before and after AuNP immobilization}

The Si/SAM samples were previously characterized by X-Ray Photoelectron Spectroscopy and contact angle measurements. ${ }^{10}$ The results confirmed the successful grafting of APTES on silicon surfaces. Upon adding MUA to the APTES-modified layers, only $24 \%$ of the amine functions reacted leading to a mixed layer $\mathrm{SH} / \mathrm{NH}_{2}(24 \%$ / 76\%). In order to gain insight into the structure of the SAM, we acquired SFG spectra by tuning the IR source over the spectral range of $\mathrm{CH}_{x}(x=$ $2,3)$ stretching modes. The molecular response $\chi_{m}^{(2)}$ becomes resonant, and its complex amplitude increases, when the IR frequency matches that of a vibration mode fulfilling two conditions: the mode must be both IR and Raman active for one allowed combination of polarizations, and must not violate symmetry rules, which essentially state that inversion symmetry leads to a vanishing $\chi_{m}^{(2)}$. This accounts for the very weak $\mathrm{CH}_{2}$ stretch signals for an ordered alkyl chain, slightly increasing when gauche defects are present. Figure 2 shows a typical spectrum for Si/APTES 
interface. This spectral region is rather crowded with vibration mode peaks (see also Figs. 3 and 4). Signals from the SAM are weak; peaks overlap and interfere with each other, as well as with a weak non-resonant contribution arising from silicon. The first step is therefore to identify SFG peaks by acquiring additional spectra. Adding the gold nanoparticles (Figure 2, bottom) increases the signalto-noise ratio, but has two consequences: firstly, a new source of $\mathrm{CH}_{x}$ (the citrate molecular layer surrounding the particles) and secondly an increased non-resonant response mainly due to gold interband transitions (see the Y-scale, approx. 10 times more intense). However, AuNPs allow for the tuning of the visible wavelength of excitation involved in the SFG process (values used are the following: $500,532,568,620$, and $670 \mathrm{~nm}$ ), leading to a phase change between resonant and non-resonant contributions (Figure 3). In this manner, we can observe dip or peak shapes for the molecular vibration modes in the experimental spectra, ${ }^{43}$ especially when the phase difference between molecular and $\mathrm{Si}+\mathrm{AuNP}$ contributions varies approximately from $0^{\circ}$ to $90^{\circ}$ together with an increase in absolute value, when changing color from red to blue as a consequence of gold response changing from weak intraband to strong interband origin. ${ }^{44}$ This greatly facilitates peak attribution as it will be stated in Table 1.

On all spectra, we observe the expected $\mathrm{CH}_{2}$ symmetric $\left(2863 \mathrm{~cm}^{-1}\right)$ and antisymmetric (2933 $\mathrm{cm}^{-1}$ ) stretching bands in the region (2800-3000 $\left.\mathrm{cm}^{-1}\right)$. The major concern is the presence, even in the spectra of Si/SAMs, of a band at higher wavelength (around $2978 \mathrm{~cm}^{-1}$ ) where the antisymmetric $\mathrm{CH}_{3}$-stretch-like resonance is normally observed. The presence of unreacted ethoxy groups in APTES layers, still under debate in the literature, may explain this observation. We therefore tried to precisely establish the origin of this band. As a matter of fact, all the observed vibrations may have three different origins: the silane layer, the organic molecules surrounding the AuNPs (when present) or some organic contaminants at the surface. The $2978 \mathrm{~cm}^{-1}$ mode is present both with and without AuNPs, and the overall lineshapes in both spectra in Figure 2 look identical. If this band was due to some contaminant, it would disappear, at least partially, as a consequence of AuNP intake, contrary to what is observed in Figure 2. Therefore, this origin is not likely. Attributing this mode to the citrates surrounding the nanoparticles would not explain the signals measured 


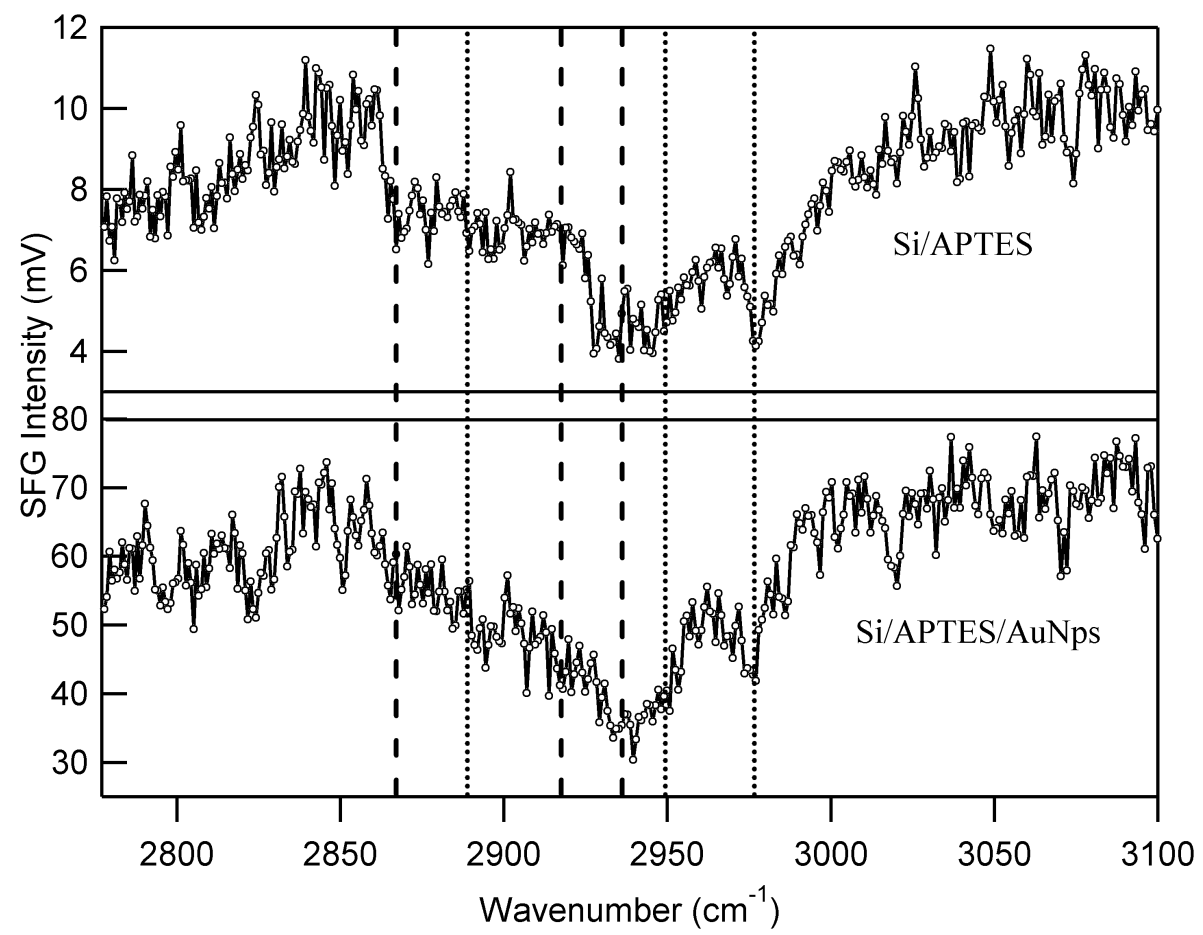

Figure 2: Typical SFG spectra recorded for APTES Si/SAM (top) and APTES Si/SAM/AuNP (bottom) at an excitation wavelength of $532 \mathrm{~nm}$.

on Si/SAM prior to AuNP adsorption. We therefore strongly favour the explanation of unreacted ethoxy groups in APTES layers. To confirm this attribution, we have performed a third set of SFG experiments, by functionalizing the particles with thiols, which remove citrate contribution and further suppress contaminants. When the $\mathrm{CH}_{3}$-terminated dodecanethiol (DDT), is grafted, the antisymmetric methyl stretch is observed at the same position as the signal from the SAM (Figure 4b). Whereas, upon thiophenol grafting (Figure 4a), the lineshape does not change between 2900 $\mathrm{cm}^{-1}$ and $3000 \mathrm{~cm}^{-1}$, and a distinctive peak from thiophenol $\left(\sim 3078 \mathrm{~cm}^{-1}\right)$ appears, indicating that citrates have been replaced as expected by thiols. ${ }^{14}$ This observation provides additional evidence that the band at $2978 \mathrm{~cm}^{-1}$ arises from unreacted ethoxy groups in the APTES SAM, which is coherent with previous findings in the literature. ${ }^{33,45,46}$ Note that this signal is very weak, suggesting that the amount of remaining $\mathrm{CH}_{3}$ is rather low and/or that these groups are poorly ordered. The ratio $\mathrm{R}$ of symmetric over antisymmetric stretching intensities is directly related to the average orientation of the $\mathrm{CH}_{3}$ groups. ${ }^{23}$ This relationship is valid for high index substrates, which favours 
electric field components along the $z$-axis (Figure 1). Given the refractive index of silicon in the visible and IR ranges, ${ }^{17,47}$ this approximation remains acceptable here. In the recorded spectra, this ratio is very small in the native SAMs, as symmetric contributions are hardly visible. This leads to favour a mean geometry of $\mathrm{CH}_{3}$ groups somewhat parallel to the surface, which is compatible with the hypothesis that some ethoxy groups remain unreacted, even in the first silane layer in contact with the silicon substrate.

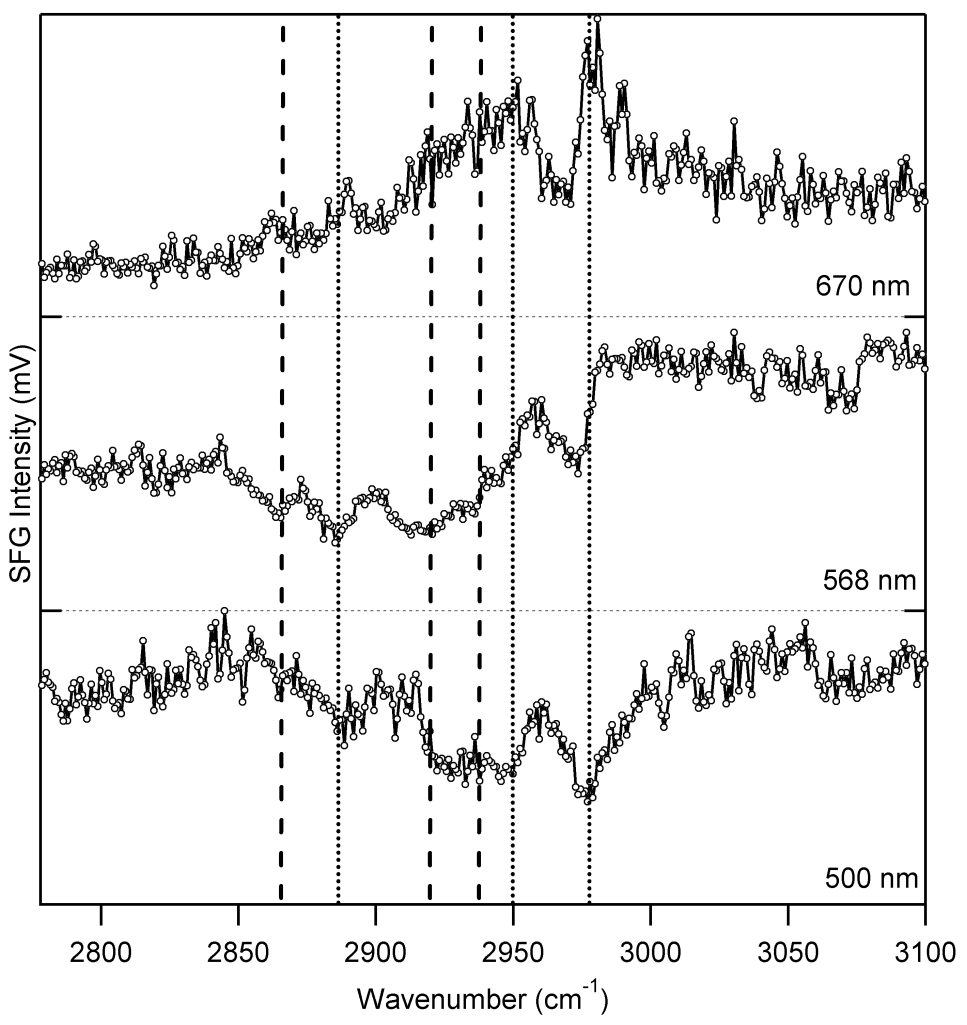

Figure 3: SFG spectra of the APTES Si/SAM/AuNP sample for different excitation wavelengths of the visible domain $(670,568$ and $500 \mathrm{~nm})$.

Having ascribed the $2978 \mathrm{~cm}^{-1}$ band to $\mathrm{CH}_{3}$ ethoxy groups, vibration mode attribution becomes easier. Using several visible wavelengths allows to first separate the various peaks (Figure 3). It is worth noting that lineshapes evolve differently with the incident visible wavelengths (Figure 3). These differences between spectra allow several resonances to appear on some spectra and not on others, as a consequence of interferences between vibration modes and the non-resonant background, ${ }^{41}$ or between neighboring vibration modes. ${ }^{42}$ We need at least three visible wave- 

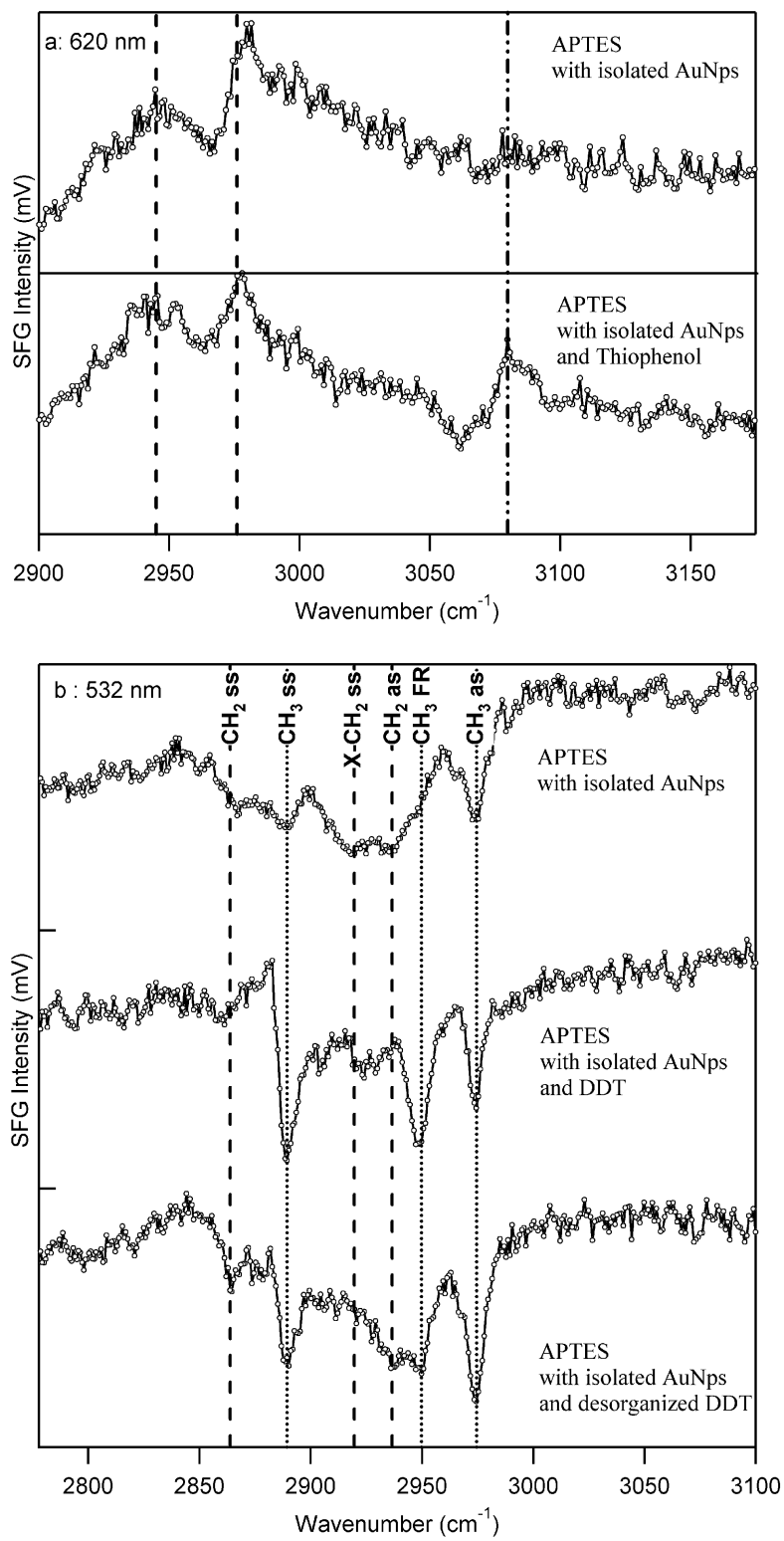

Figure 4: (a) SFG spectra for different samples at $620 \mathrm{~nm}$ and in the spectral range of 2900-3175 $\mathrm{cm}^{-1}$ for one sample with APTES and isolated AuNPs without (up) and with (down) thiophenol molecules. (b) in the spectral range of $2780-3100 \mathrm{~cm}^{-1}$ at $532 \mathrm{~nm}$. Absolute scales vary from one spectrum to another. 
lengths to identify six vibration modes as depicted in Figures 3 and 4.

Table 1: SFG band attribution table for Si/APTES and Si/APTES/AuNP interfaces.

\begin{tabular}{ll}
\hline Vibration position $\left(\mathrm{cm}^{-1}\right)$ & Vibration mode \\
\hline 2863 & $\mathrm{CH}_{2}$, ss \\
2888 & $\mathrm{CH}_{3}$, ss \\
2911 & $\mathrm{X}-\mathrm{CH}_{2}$, ss \\
2933 & $\mathrm{CH}_{2}$, as \\
2945 & $\mathrm{CH}_{3}, F R$ \\
2978 & $\mathrm{CH}_{3}$, as \\
\hline
\end{tabular}

In order to attribute each mode, we used a differential approach by comparing native Si/SAM to $\mathrm{Si} / \mathrm{SAM} / \mathrm{AuNP}$ and Si/SAM/AuNP/DDT samples (Figures 3 and 4), in addition to data from the literature $^{48,49}$ (see Table 1). In particular, we measured the SFG spectrum of a Si/SAM/AuNP/DDT sample left for two days in air, which induces some disorder in the layer, and therefore enhances the alkyl $\mathrm{CH}_{2}$ response, helping to attribute $\mathrm{CH}_{2}$ modes. The two vibrations located at $2863 \mathrm{~cm}^{-1}$ and $2933 \mathrm{~cm}^{-1}$ are ascribed to the symmetric and asymmetric $\mathrm{CH}$ stretching vibration modes of methylene with the carbon surrounded by two other carbon atoms. ${ }^{50,51}$ On the contrary, the vibration located at $2911 \mathrm{~cm}^{-1}$ corresponds to symmetric $\mathrm{CH}$ stretching vibration mode of methylene with the carbon linked to a heteroatom ( $\mathrm{Si}$ or $\mathrm{N}$ in this case, denoted $\left.\mathrm{X}-\mathrm{CH}_{2}\right) .{ }^{48}$ The modes located at 2888, 2978 and $2945 \mathrm{~cm}^{-1}$ are related to symmetric (ss), asymmetric (as) and Fermi resonance (FR) $\mathrm{CH}$ stretching vibration modes of $\mathrm{CH}_{3}$ methyl groups. In fact, the three close vibration modes $\left(2911,2933\right.$, and $\left.2945 \mathrm{~cm}^{-1}\right)$ strongly interfere together, which makes their attribution difficult based solely on the spectra in Figure 2.

\section{Characterization of the gold nanoparticle deposition}

AuNP were deposited on two chemical layers: APTES or APTES modified by MUA (see Figure 1). The dipping time in AuNP solution was varied, either 30 or 60 minutes, to change the surface coverage. To ensure the reproducibility of our method, we repeated the protocol three times for each sample. AuNP surface coverage was determined by SEM. For the two Si/SAM, APTES and APTES/MUA, two types of surface coverage were observed: isolated (Figures 5a, 5c, 5e, 5g) 
and coupled (Figures 5b, 5f, 5d, 5h) AuNPs. Based on AuNP coverages, samples were arranged into four categories (denoted A-I and A-C for isolated and coupled AuNPs on APTES, and M-I and M-C for isolated and coupled AuNPs on APTES/MUA. See supplementary information for SEM images and classification). Figure 5 shows typical SEM images and absorption spectra for each family (represented by the member with the highest surface density). Surface coverage and AuNP layer homogeneity measurements were performed in several regions of the samples. We determined from SEM images the average surface density of AuNPs $(\rho)$ and the corresponding mean center-to-center distance between particles $\mathrm{D}_{m}=\rho^{-1 / 2}$. Results are summarized in Table 2 for the four samples of Figure 5. As expected, increasing dipping time led to higher surface coverage, regardless of the nature of the SAM. After 30 minutes (Figure 5a), gold nanoparticles are well separated whereas after 60 minutes (Figures 5b, 5c, 5d), the nanoparticles come closer to each other on the substrate and eventually interact (the distance between AuNP is in the same range as their size, Figure 5d) or even aggregate in small clusters (Figure 5b). The reproducibility depends on the nature of the SAM. Using APTES, the samples are rather poorly reproducible and fall into the A-I (1/3) and A-C (2/3) categories; the average surface density is high (more than $5.10^{10}$ AuNPs. $\mathrm{cm}^{-2}$ ); dispersion is homogeneous. For high densities, small clusters are present over the entire surface (Figure 5b); aggregates exist even for low densities. For APTES/MUA samples, reproducibility is better with only $1 / 6$ falling into the M-C category and 5/6 into the M-I category (see Figure 5d). The average surface coverage is lower but dispersion is more homogeneous than for APTES samples; high densities lead to particles interacting by being close together, but the number of clusters remains low; no aggregation is observed for low densities. We note that, for some samples, there is coexistence of isolated and clustered areas on various spots of the surface; these were assigned to a category based on their dominant character (Figure 5).

We performed UV-visible measurements at $55^{\circ}$ incidence on the same set of samples in reflection for $s$ and $p$ polarisations. The typical responses of four representative samples are shown in Figures 5e, 5f, 5g, 5h. For isolated nanoparticles, the A-I and M-I categories, we observe a unique contribution around $505 \mathrm{~nm}$ for both $p$ and $s$ polarisations. This peak $(p)$ or dip $(s)$ originates 

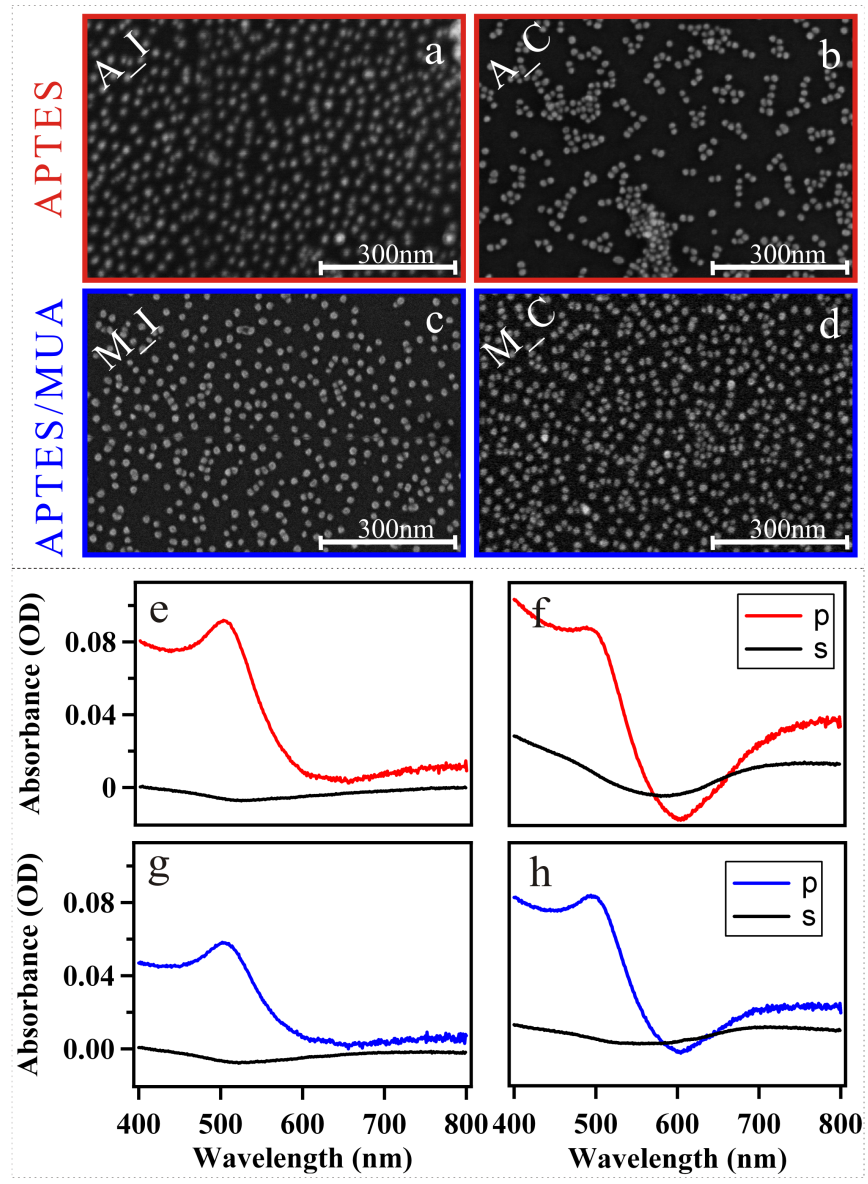

Figure 5: SEM images of silicon surfaces after nanoparticle deposition on APTES (red, top images) or APTES/MUA (blue, bottom images) for isolated AuNPs (left: a, c) and coupled AuNPs (right: b, d); UV-visible measurements of silicon surfaces after nanoparticle deposition on APTES (red, top graphs) or APTES/MUA (blue, bottom graphs) for isolated AuNPs (left: e, g) and coupled AuNPs (right: $f, h)$.

from the localized surface plasmon resonance of AuNPs (LSPR), and its wavelength corresponds to nanoparticles in air as described by the dipolar approximation. The peak or dip shapes are explained by the differences in refractive indices between the silicon substrate and the adsorbed layers. ${ }^{22}$ For coupled nanoparticle layers, the A-C and M-C categories, a second feature appears as a pronounced dip around $600 \mathrm{~nm}$, followed by a rise in absorbance for higher wavelengths, clearly visible with $p$-polarized light. This contribution is due to the aggregation of particles as a consequence of longitudinal coupling. ${ }^{13}$ In order to establish a correlation between UV-visible spectra in $p$-polarized light and AuNP surface coverage, we correlated two absorbance amplitudes to the AuNP coverage: A 505 , corresponding to isolated AuNPs and measured by the difference between 
Table 2: Surface density and mean distance between particles for each category of samples (DT = Dipping Time, $\mathrm{SC}=$ Sample Category).

\begin{tabular}{lllll}
\hline Silane & DT $(\min )$ & SC & $\rho\left(10^{10}{\left.\mathrm{AuNPs} . \mathrm{cm}^{-2}\right)}_{1} \mathrm{D}_{m}(\mathrm{~nm})\right.$ \\
\hline APTES & 30 & A-I & $9.86 \pm 0.05$ & $31.8 \pm 0.1$ \\
APTES & 60 & A-C & $11.6 \pm 0.1$ & $29.3 \pm 0.1$ \\
APTES/MUA & 60 & M-I & $9.63 \pm 0.06$ & $32.2 \pm 0.1$ \\
APTES/MUA & 60 & M-C & $14.1 \pm 0.1$ & $26.6 \pm 0.1$ \\
\hline
\end{tabular}

the local maximum $(505 \mathrm{~nm})$ and minimum, as depicted in Figure 6a, and the contribution of the interacting particles, $\mathrm{A}_{800}$, measured as the difference in absorbance between the value at $800 \mathrm{~nm}$ and the minimum. In Figures $6 \mathrm{~b}$ and $6 \mathrm{c}$, these absorbance intensities are plotted as a function of the AuNP coverage. When AuNPs are isolated (A-I and M-I categories), $\mathrm{A}_{800}$ is very low (less than $10^{-2}$ ), whereas when coupling appears (for A-C and M-C), its intensity increases.

Below the value of $10^{-2}$ for $A_{800}$, all the samples can be associated with the isolated category. Above the value of $2.10^{-2}$ for $A_{800}$ all the samples can be classified in the clustered/coupled category. Between these two limits, the behaviour of the samples shows a mixed character between isolated and interacting particles, depending on the zones probed by SEM. Partial aggregation may occur for rather low densities with APTES SAM, but only for the highest one with MUA (threshold around $\rho \approx 10^{11}$ AuNPs. $\mathrm{cm}^{-2}$ ). On the contrary, $\mathrm{A}_{505}$ increases approximately continuously with AuNP coverage, regardless of the aggregation state. Of course, heterogeneous dispersion induces a broad distribution of surface coverage values, which has an impact on the accuracy of the $x$-axis values in Figures $6 \mathrm{a}, 6 \mathrm{~b}, 6 \mathrm{c}$. The following shows that it is nevertheless possible to quantitatively analyze parameter $A_{505}$. To this end, we fit $A_{505}$ as a function of $D_{m}$ (Figure $6 \mathrm{~d}$ ). In this case, the relative uncertainty on the $x$-axis is divided by 3 , making the adjustment more appropriate. The best fit is obtained using a power law, with an exponent close to -2 . This result confirms the linear fit of $\mathrm{A}_{505}$ with respect to the surface density $\rho$. Recording a full UV-visible spectrum takes less than 5 minutes, whereas a full SEM analysis requires more than one hour, since several images must be recorded for each sample, then analyzed in order to estimate a density. The relationships shown here provide a fast and easy way to estimate the quality of a set of samples by UV-Visible spectroscopy. 

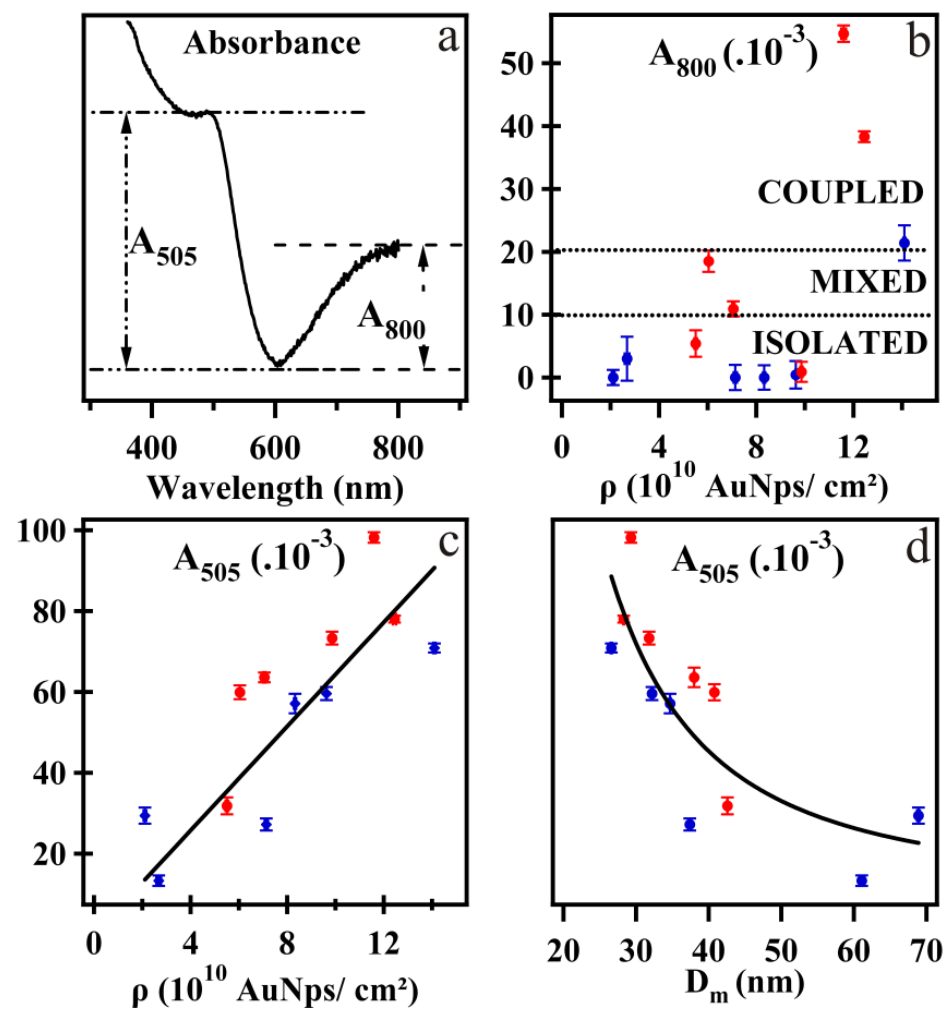

Figure 6: (a) Definition of $\mathrm{A}_{505}$ and $\mathrm{A}_{800}$. (b) Absorbance amplitude at $800 \mathrm{~nm}$ (blue for APTES/MUA and red for APTES) versus surface density $\rho$. The coupled, mixed and isolated characterization refers to the surface distribution of nanoparticles and is deduced from the SEM images. (c) Absorbance amplitude at $505 \mathrm{~nm}$ (blue for APTES/MUA and red for APTES) versus surface density $\rho$. (d) Absorbance amplitude at $505 \mathrm{~nm}$ versus mean particle distance $\mathrm{D}_{m}=\rho^{-1 / 2}$.

\section{Stability in time}

We monitored the stability in time of the AuNP layers by UV-Visible measurements. We prepared Si/SAM/AuNP samples with APTES and APTES/MUA SAMs, and recorded their UV-Visible spectra immediately after gold grafting (Figure 7). They were then stored in air-tight boxes in the dark and at room temperature for several days. Then, the aged substrates were functionalized by an alkanethiol and analyzed for several hours on the SFG set-up (i.e. exposed for several hours to short, high intensity visible and infrared pulses). The samples were then checked on the UV-visible spectrophotometer several times over the following three months, and eleven months later. Figure 7 shows that the samples remain very stable over this eleven month period in spite of the chemical and optical degradation they sustained. The APTES+MUA material (Figure 7a) shows no sign 
of ageing (except for an insignificant baseline drift, intrinsic to the spectrophotometer alignment, which is supressed by imposing a null minimum for each spectrum) after several months, and a small decrease for very long periods of time. As for the APTES SAM, a decrease of the $\mathrm{A}_{505}$ signal was observed over time, characteristic of the presence of the gold nanoparticles. Some of the particles (isolated and aggregated) may have been removed from the surface when the sample was handled or characterized. Nevertheless, the layers of isolated particles seem to remain quite stable over time. Moreover, if the sample suffers no degradation (see insert of Figure 7b), its absorbance remains identical even after eleven months. We may therefore conclude that these grafting methods enable the immobilization of a high density of AuNPs on silicon samples with a high stability.
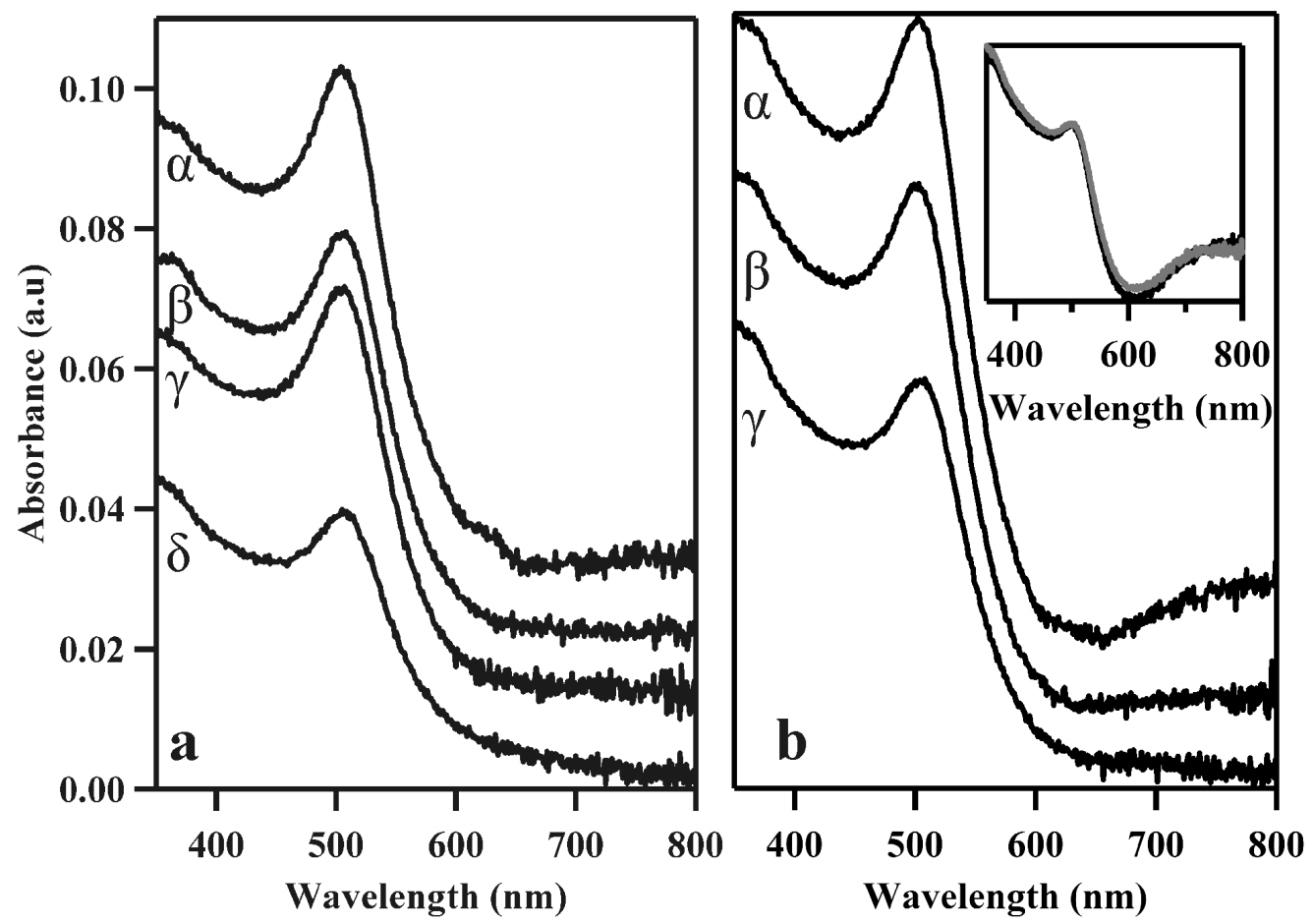

Figure 7: UV-visible spectra of two samples with (a) and without MUA (b) for 30 minutes dipping times at different experimental steps. For APTES/MUA: $\alpha$ : immediately after preparation, $\beta$ : several days after preparation and with dodecanethiol functionalization, $\gamma$ : several days later and exposed to laser sources for 15 hours, $\delta$ : eleven months later. For APTES: $\alpha$ : immediately after preparation, $\beta$ : several weeks after preparation, exposed to laser sources for 10 hours, with thiophenol functionalization, and exposed again to the laser sources for 15 hours, $\gamma$ : eleven months later. Insert, sample with APTES immediately after preparation (black) and eleven months later (grey) with no laser exposure or functionalization. Each curve is shifted by 0.01 from the previous one for clarity. 


\section{Effect of gold nanoparticles on the intensity of the SFG signal}

For the four samples described in Figure 5 (each representing one of the categories, A-I, A-C, M-I and M-C), SFG spectra were recorded between $2780 \mathrm{~cm}^{-1}$ and $3330 \mathrm{~cm}^{-1}$ (Figure 8) at the visible wavelength of $670 \mathrm{~nm}$ (see also SI section for other visible wavelengths). Spectra were normalized by absolute SFG intensities emitted by samples, because we cannot directly compare absolute SFG intensities from the samples when their compositions and structures differ. ${ }^{22}$ The $670 \mathrm{~nm}$ wavelength was chosen because the vibrations appear mostly as peaks with a rather high intensity and, because it makes it possible to monitor the differences between isolated and aggregated AuNP layers. Four vibrations among the six listed in Table 1 are clearly observed on all samples, but the central region is too crowded to observe the missing $\mathrm{CH}_{2}$ modes. The four samples exhibit an essentially uniform vibrational response, with two small differences: the intensities of $\mathrm{CH}_{2}$ vibration modes are higher for APTES/MUA SAMs, which stems from the additional methylene functions from the MUA. The $\mathrm{CH}_{3}$ symmetric response is also a bit more intense, but at this stage it would seem to be a structural feature intrinsic to the SAM rather than a consequence of the gold grafting. To a first order approximation, the vibrational features before and after grafting of gold may be considered identical (see also Figure 2). Probing the samples in different points on their surfaces essentially produces the same results. This fact reinforces the hypothesis that the unreacted ethoxy groups from APTES are buried below the surface, are not significantly affected by the MUA post-modification, and do not participate in the gold grafting. More information could be gained from the monitoring of the end groups of the silane chains. Previous studies on alkylsilanes have shown that even the modifications of $\mathrm{CH}_{3}$ moieties induced by interaction with deposited molecules or cells are rather small, but measurable. ${ }^{52-54}$ In our case, we have verified experimentally that the SFG activity of terminal $\mathrm{NH}_{2}$ groups in the $\mathrm{NH}$ stretching region is too weak to be measured effectively, which is consistent with its low infrared and Raman activities. ${ }^{51}$ Some SFG measurements indicate that this mode only becomes active after curing the APTES SAM at high temperature. ${ }^{33}$

The major difference between the SFG spectra lies in the absolute intensities of the vibrations 


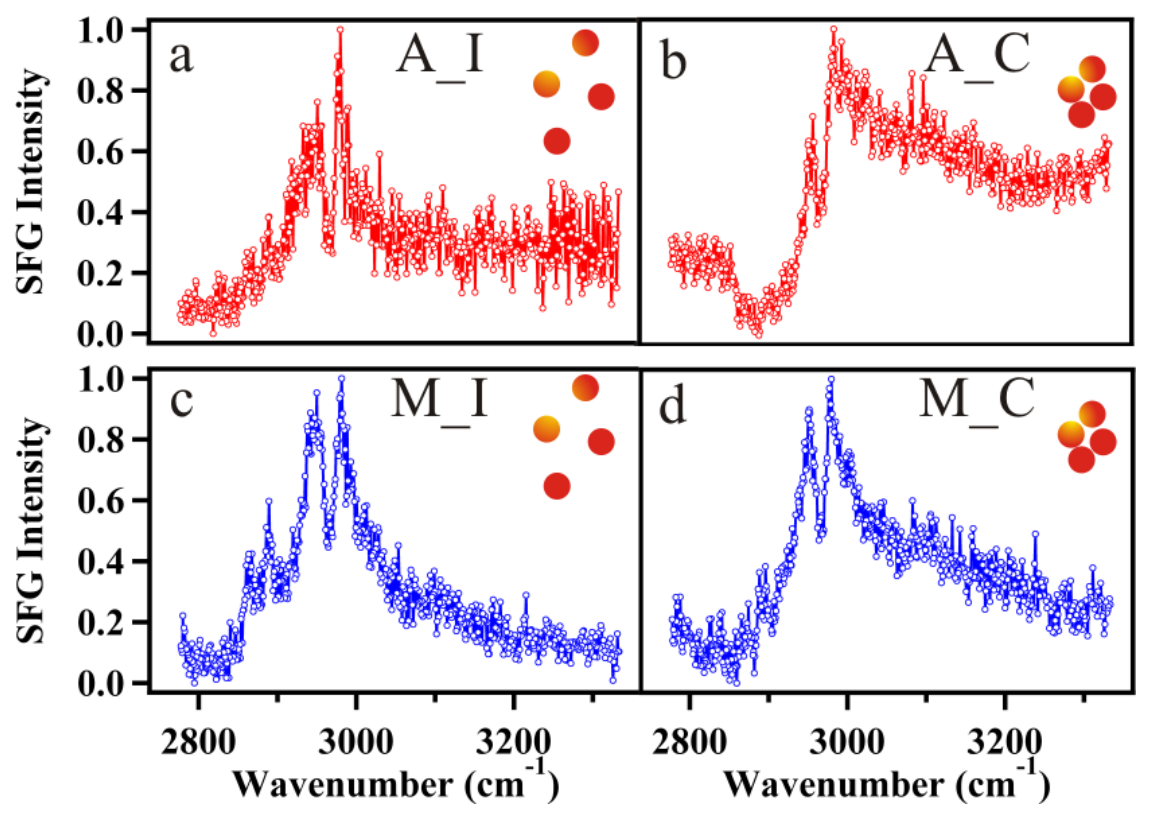

Figure 8: SFG spectra for an incident wavelength of $670 \mathrm{~nm}$ for APTES (red) and APTES/MUA (blue) where AuNPs are isolated $(\mathrm{a}, \mathrm{c})$ or coupled $(\mathrm{b}, \mathrm{d})$. All spectra are normalized to 1 in order to compare the different lineshapes.

and of the non-resonant background (originating from the $\chi_{S i / A u}^{(2)}$ term as explained in the introduction). These contributions are small at $670 \mathrm{~nm}$ but nonetheless result in various lineshapes and interference patterns with vibration modes. Direct observation of SFG data shows that particle aggregation leads to an enhancement of the $\mathrm{Si}+\mathrm{Au}$ background. In order to quantify this phenomenon, we must evaluate the different relevant contributions. We therefore measured the ratio of non-resonant to resonant intensities (NR/R), as directly extracted from the raw spectra. This helps to avoid several problems resulting from comparing absolute SFG intensities between different spectra: the reproducibility from sample to sample; the stability of the power, size and focus of the incident beams; changes in the Fresnel factors and local field amplifications from the variability in composition and structure of the AuNP layer. The maximum intensity of the antisymmetric $\mathrm{CH}_{3}$ peak represents the resonant contribution, whereas the non-resonant contribution is calculated using the average value of the SFG intensity at $3100 \mathrm{~cm}^{-1}$, i.e. far from any molecular resonance (Table 3). 
Table 3: NR/R ratio for the $670 \mathrm{~nm}$ excitation and surface density for the four samples in figure 8 .

\begin{tabular}{lllll}
\hline Sample & $\left(\mathrm{A}_{505}\right)^{2}$ & $\rho\left(10^{10}\right.$ AuNPs. $\left.\mathrm{cm}^{-2}\right)$ & $\rho^{2}$ & NR/R \\
\hline A-I & 0.537 & 9.86 & 97.22 & 0.308 \\
A-C & 0.964 & 11.6 & 135.49 & 0.636 \\
M-I & 0.355 & 9.63 & 92.74 & 0.201 \\
M-C & 0.502 & 14.1 & 150.06 & 0.413 \\
\hline
\end{tabular}

Consistent with our data, we can make the following approximation: considering that constant factors in the SFG intensity will disappear after dividing the $\mathrm{Si}+\mathrm{Au}$ intensity by the resonant intensity, the $\mathrm{Si}+\mathrm{Au}$ intensity is proportional to the square of the nanoparticle surface density $(\rho)$, and the resonant intensity to the square of the surface density of ethoxy groups $\left(\rho_{\text {mol }}\right)$. The ratio NR/R should therefore be proportional to $\left(\rho / \rho_{m o l}\right)^{2}$. Considering that the structures of the ethoxy groups in all samples remain roughly constant as shown above, we hypothesize that $\rho_{m o l}$ is also essentially constant in all of the APTES layers, modified or not by MUA. Therefore, proportionality between NR/R and $\rho^{2}$ should be visible in Table 3, which is not the case. However, both parameters $A_{505}$ and $\rho$ are an expression of the surface density of nanoparticles. They incorporate the rather high surface heterogeneity into one macroscopic parameter. The discussion above shows that they perform it in a correlated way, but with strong differences due to their specificities. Calculated based on electron microscopy images, $\rho$ is the average value, in the various zones investigated on the surface, of a purely local parameter, for which the distribution function is at least as interesting as its mean value. $\mathrm{A}_{505}$ on the contrary is a macroscopic quantity, which directly integrates the various local values of surface density over the whole surface of the light spot. Thus, $\mathrm{A}_{505}$, seems more appropriate to quantify an effective surface density in an optical experiment. Figure 9 confirms that the NR/R ratio is in fact proportional to $\left(\mathrm{A}_{505}\right)^{2}$.

This point is worth noting, since we have observed in past studies that it is difficult to establish the proportionality between the SFG intensities and the square of the surface nanoparticle density. We have shown that taking into account the reflectivity effect (Fresnel factors ${ }^{55}$ ) in the SFG experiment produces results that are in better agreement with this law. ${ }^{17}$ Nevertheless, data show that this proportionality is sometimes rather approximate. ${ }^{14}$ Here, we propose an alternate method, and 
show that the average surface density measured by SEM (or AFM) is too sensitive to fluctuations on the surface on a macroscopic scale. On the contrary, the $A_{505}$ parameter is a first step in the integration of this surface heterogeneity and therefore constitutes a more reliable value of the actual density. In addition, as an optical measurement, $\mathrm{A}_{505}$ already encompasses the reflectivity contributions, making the link to SFG intensities more straightforward. Given the rather wide variety of surface distributions encountered in the set of films studied here, it is quite useful to have access to a single, easily measurable quantity, $\mathrm{A}_{505}$, which accurately describes the state of the AuNP layer.

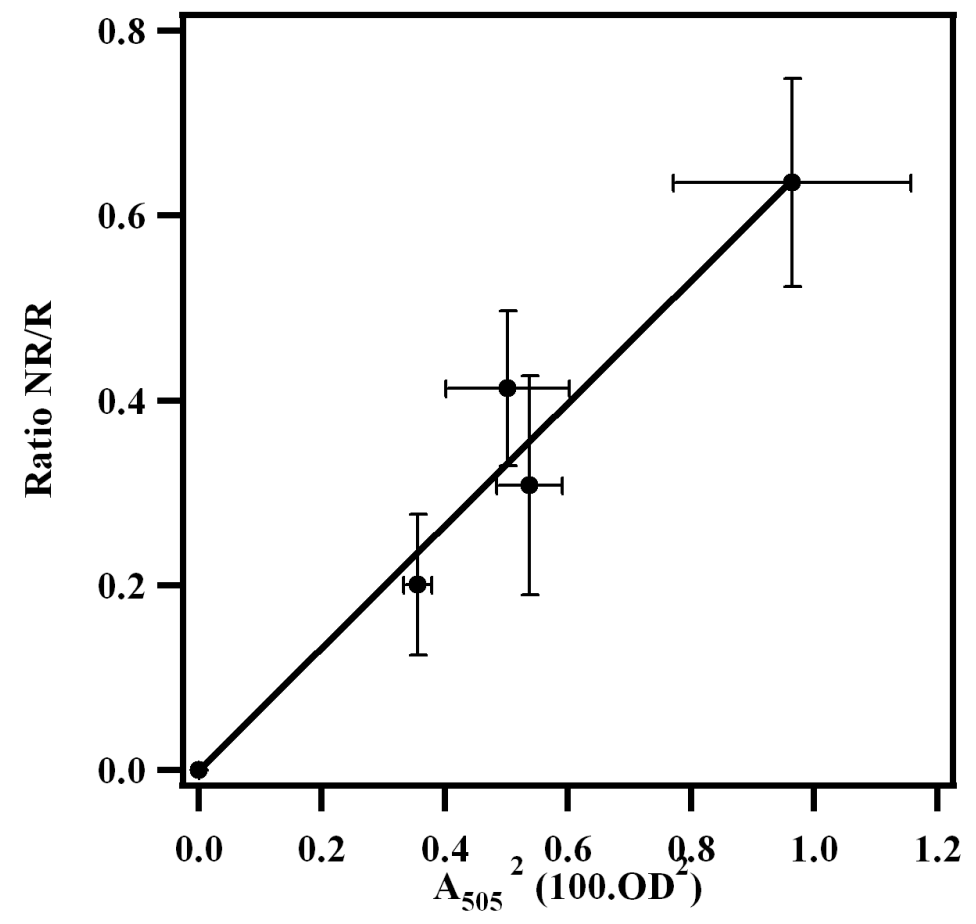

Figure 9: Ratio NR/R plotted as a function of $\left(\mathrm{A}_{505}\right)^{2}$.

Finally, the proportionality shown in Figure 9 is additional evidence that the SFG response, both molecular and non-resonant, is enhanced by the plasmonic properties of the grafted gold particles. On the other hand, aggregation seems to have almost no effect on this enhancement. This would seem to contradict the spectra in Figure 8, in which the apparent rise of the NR signal of the A-C sample is essentially due to the high concentration of particles at the surface. This can be explained by the properties of SFG spectroscopy. As stated above, due to the high refractive index of silicon, SFG in a ppp polarization scheme mostly monitors the $(z z z)$ coefficient of $\chi^{(2)}$, where $z$ 
is the axis perpendicular to the surface ${ }^{17}$ As a consequence, the plasmonic properties involved in SFG enhancement mostly derive from perpendicular surface plasmons. When aggregation occurs, longitudinal coupling between particles strongly affects the in-plane plasmonic properties, whereas the perpendicular ones are only modified through transverse coupling, which in principle results in a weak blueshift and damping, ${ }^{56}$ all these effects being included in the $\mathrm{A}_{505}$ parameter. However, a detailed study on the effect of aggregation on enhancement factors requires fitting spectra recorded for several visible wavelengths, in order to fully separate the molecular and non-resonant contributions in an absolute manner. This systematic analysis is planned for future studies.

\section{Conclusion}

In this article, we investigated Si/SAM/AuNP films for different surface coverages of gold nanoparticles and two SAMs, APTES and APTES/MUA. We combined microscopic and spectroscopic tools for the films analysis. SFG analysis of the APTES and APTES/MUA SAMs allowed us to

identify all the corresponding vibration modes. In spite of the weakness of the SFG signals, this spectroscopy showed the presence of unreacted ethoxy groups from the silane. These terminal $\mathrm{CH}_{3}$ functions lie mostly parallel to the silicon plane. AuNP grafting led to a significant enhancement of the SFG signal but did not modify the internal structure of the SAM. The local enhancement of SFG signals made it possible to monitor the weak response of our samples by tuning the visible wavelength involved in the SFG process. Using APTES or APTES modified by MUA as an interlayer between silicon and AuNPs led to significant differences. The addition of MUA stabilized the gold nanoparticle layer and favoured homogeneous adsorption of isolated particles up to high surface coverages. On the contrary, APTES alone led to less organized layers, which even at medium surface coverages showed the presence of aggregates. Both grafting methods showed excellent stability over long periods of time, proving the usefulness of these high quality layers for further applications. If the goal is to adsorb as many particles as possible while ensuring that they remain isolated, then the best choice is APTES/MUA grafting, provided that the surface density remains 
below $10^{11}$ particles per $\mathrm{cm}^{2}$. On the contrary, if the key parameter is the local density of particles, and not the structure of the AuNP layer, then APTES and APTES/MUA SAMs exhibit rather similar behaviours, while ageing films, surprisingly, seems to improve the quality of the APTES samples. The structure of the AuNP layer was investigated by electron microscopy and visible reflectance spectroscopy. Microscopy images provide useful information on the heterogeneity of the particle dispersion on the surface, but they are inadequate for calculating an average surface coverage. An effective value of this quantity is better estimated through the amplitude of the plasmon resonance of isolated particles, which is shown to vary proportionally to the surface density measured by microscopy, but also includes heterogeneity effects in a single measurement. In addition, the aggregation state of grafted AuNPs shown by microscopy images is in the same way quantified by the amplitude of the longitudinally coupled surface plasmon resonance in reflectance spectra, providing a fast quantitative criterion for distinguishing isolated and aggregated films. Finally, we observed that for SFG spectroscopy of the SAM layer, the key parameter is indeed the local density of particles, whether aggregated or not. The various relative intensities of molecular and non-resonant contributions to the SFG spectrum are due to a weak amplification of the SFG process due to the coupling to plasmonic properties of the AuNPs, while the $\mathrm{Si}+\mathrm{Au}$ contribution is in addition proportional to the square density of particles, as measured by visible reflectance spectroscopy rather than by microscopy. The best compromise is to be found between an efficient amplification for future spectroscopic studies of molecules adsorbed at the surface of the particles (and not of the underlying SAM). The aggregated or isolated character of the nanoparticles may however become important, especially for the generation of hot spots between two particles which would produce additional enhancements.

\section{Acknowledgement}

This research was supported by the Région Ile-de-France as part of the C'Nano IdF funding program under the CREMOSOFT grant agreement. The authors also acknowledge the ERM financial support of the Université Paris-Sud. We thank Dr. A. Hervier for fruitful scientific discussions. 


\section{Supporting Information Available}

This material is available free of charge via the Internet at http://pubs.acs.org.

\section{References}

(1) Anker, J. N.; Hall, W. P.; Lyandres, O.; Shah, N. C.; Zhao, J.; Van Duyne, R. Biosensing with Plasmonic Nanosensors. Nat. Mater. 2008, 7, 442-453.

(2) Sardar, R.; Funston, A. M.; Mulvaney, P.; Murray, R. W. Gold Nanoparticles: Past, Present, and Future. Langmuir 2009, 25, 13840-13851.

(3) Stewart, M. E.; Anderton, C. R.; Thompson, L. B.; Maria, J.; Gray, S. K.; Rogers, R. G.; Nuzzo, R. G. Nanostructured Plasmonic Sensors. Chem. Rev. 2008, 108, 494-521.

(4) Bishop, L. M.; Tillman, A. S.; Geiger, F. M.; Haynes, C. L.; Klaper, R. D.; Murphy, C. J.; Orr, G.; Pedersen, J. A.; De Stefano, L.; Hamers, R. J. Enhancing Graduate Student Communication to General Audiences through Blogging about Nanotechnology and Sustainability. J. Chem. Educ. 2014, 91, 1600-1605.

(5) Link, S.; El-Sayed, M. A. Spectral Properties and Relaxation Dynamics of Surface Plasmon Electronic Oscillations in Gold and Silver Nanodots and Nanorods. J. Phys. Chem. B 1999, $103,8410-8426$.

(6) Pelaz, B.; del Pino, P. In Frontiers of Nanoscience; Elsevier, 2012.

(7) Liu, X. H.; Wang, Y.; Chen, P.; Wang, Y. S.; Mang, J. L.; Aili, D.; Liedberg, B. Biofunctionalized Gold Nanoparticles for Colorimetric Sensing of Botulinum Neurotoxin A Light Chain. Anal. Chem. 2014, 86, 2345-2352.

(8) Morel, A. -L.; Boujday, S.; Methivier, C.; Krafft, J. -M.; Pradier, C. -M. Biosensors Elaborated on Gold Nanoparticles, a PM-IRRAS Characterisation of the IgG Binding Efficiency. Talanta 2011, 85, 35-42. 
(9) Morel, A. -L.; Volmant, R. -M.; Methivier, C.; Krafft, J. -M.; Boujday, S.; Pradier, C. -M. Optimized Immobilization of Gold Nanoparticles on Planar Surfaces through Alkyldithiols and their Use to Build 3D Biosensors. Colloid Surf. B-Biointerfaces 2010, 81, 304-312.

(10) Ben Haddada, M.; Blanchard, J.; Casale, S.; Krafft, J. -M.; Vallee, A.; Methivier, C.; Boujday, S. Optimizing the Immobilization of Gold Nanoparticles on Functionalized Silicon Surfaces: Amine- vs Thiol-Terminated Silane. Gold Bull. 2013, 46, 335-341.

(11) Lundgren, A.; Hulander, M.; Brorsson, J.; Hermansson, M.; Elwing, H.; Andersson, O.; Liedberg, B.; Berglin, M. Gold-Nanoparticle-Assisted Self-Assembly of Chemical Gradients with Tunable Sub-50 nm Molecular Domains. Part. Part. Syst. Charact. 2014, 31, 209-218.

(12) Nath, N.; Chilkoti, A. A Colorimetric Gold Nanoparticle Sensor To Interrogate Biomolecular Interactions in Real Time on a Surface. Anal. Chem. 2002, 74, 504-509.

(13) Grabar, K. C.; Freeman, R. G.; Hommer, M. B.; Natan, M. J. Preparation and Characterization of Au Colloid Monolayer. Anal. Chem. 1995, 67, 735-743.

(14) Humbert, C.; Pluchery, O.; Lacaze, E.; Tadjeddine, A.; Busson, B. Optical Spectroscopy of Functionalized Gold Nanoparticles Assemblies as a Function of the Surface Coverage. Gold Bull. 2013, 46, 299-309.

(15) Liu, Y.; Yehl, K.; Narui, Y.; Salaita, K. Tension Sensing Nanoparticles for Mechano-Imaging at the Living/Nonliving Interface. J. Am. Chem. Soc. 2013, 135, 5320-5323.

(16) Rahma, H.; Buffeteau, T.; Belin, C.; Le Bourdon, G.; Degueil, M.; Bennetau, B.; Vellutini, L.; Heuze, K. Resolving the Chemical Nature of Nanodesigned Silica Surface Obtained via a Bottom-Up Approach. ACS Appl. Mater. Interfaces 2013, 5, 6843-6849.

(17) Humbert, C.; Pluchery, O.; Lacaze, E.; Tadjeddine, A.; Busson, B. A Multiscale Description of Molecular Adsorption on Gold Nanoparticles by Nonlinear Optical Spectroscopy. Phys. Chem. Chem. Phys. 2012, 14, 280-289. 
(18) Nie, S. M.; Emery, S. R. Probing Single Molecules and Single Nanoparticles by SurfaceEnhanced Raman Scattering. Science 1997, 275, 1102-1106.

(19) Hoffmann, F. M. Infrared Reflection-Absorption Spectroscopy of Adsorbed Molecules. Surface Science Reports 1983, 3, 107,109-192.

(20) Aissaoui, N.; Bergaoui, L.; Landoulsi, J.; Lambert, J. -F.; Boujday, S. Silane Layers on Silicon Surfaces: Mechanism of Interaction, Stability, and Influence on Protein Adsorption. Langmuir 2012, 28, 656-665.

(21) Liu, H. -B.; Venkataraman, N. V.; Bauert, T. E.; Textor, M.; Xiao, S. -J. Multiple Transmission-Reflection Infrared Spectroscopy for High-Sensitivity Measurement of Molecular Monolayers on Silicon Surfaces. J. Phys. Chem. A 2008, 112, 12372-12377.

(22) Pluchery, O.; Humbert, C.; Valamanesh, M.; Lacaze, E.; Busson, B. Enhanced Detection of Thiophenol Adsorbed on Gold Nanoparticles by SFG and DFG Nonlinear Optical Spectroscopy. Phys. Chem. Chem. Phys. 2009, 11, 7729-7737.

(23) Bourguignon, B.; Zheng, W.; Carrez, S.; Ouvrard, A.; Fournier, F.; Dubost, H. Deriving the Complete Molecular Conformation of Self-Assembled Alkanethiol Molecules from SumFrequency Generation Vibrational Spectra. Phys. Rev. B 2009, 79, 125433.

(24) Himmelhaus, M.; Eisert, F.; Buck, M.; Grunze, M. Self-Assembly of n-Alkanethiol Monolayers. A Study by IR-Visible Sum Frequency Spectroscopy (SFG). J. Phys. Chem. B 2000, $104,576-584$.

(25) Richter, L. J.; Yang, C. S. C.; Wilson, P. T.; Hacker, C. A.; van Zee, R. D.; Stapleton, J. J.; Allara, D. L. Optical characterization of Oligo(phenylene-ethynylene) Self-Assembled Monolayers on Gold. J. Phys. Chem. B 2004, 108, 12547-12559.

(26) Guyot-Sionnest, P.; Shen, Y. R. Bulk Contribution in Surface 2nd-Harmonic Generation. Phys. Rev. B 1988, 38, 7985-7989. 
(27) Lambert, A. G.; Neivandt, D. J.; McAloney, R. A.; Davies, P. B. A Protocol for the Reproducible Silanization of Mica Validated by Sum Frequency Spectroscopy and Atomic Force Microscopy. Langmuir 2000, 16, 8377-8382.

(28) Yang, Y. J.; Hu, S. The Deposition of Highly Uniform and Adhesive Nanocrystalline PbS Film from Solution. Thin Solid Films 2008, 516, 6048-6051.

(29) Tyrode, E.; Liljeblad, J. F. D. Water Structure Next to Ordered and Disordered Hydrophobic Silane Monolayers: A Vibrational Sum Frequency Spectroscopy Study. J. Phys. Chem. C 2013, 117, 1780-1790.

(30) Weber, J.; Balgar, T.; Hasselbrink, E. Conformational Disorder in Alkylsiloxane Monolayers at Elevated Temperatures. J. Chem. Phys. 2013, 139, 244902.

(31) Wang, H.; Sapi, A.; Thompson, C. M.; Liu, F.; Zherebetskyy, D.; Krier, J. M.; Carl, L. M.; Cai, X.; Wang, L. -W.; Somorjai, G. A. Dramatically Different Kinetics and Mechanism at Solid/Liquid and Solid/Gas Interfaces for Catalytic Isopropanol Oxidation over SizeControlled Platinum Nanoparticles. J. Am. Chem. Soc. 2014, 136, 10515-10520.

(32) Uosaki, K.; Noguchi, H.; Yamamoto, R.; Nihonyanagi, S. Interfacial Molecular Structures of Polyelectrolyte Brush in Contact with Dry Nitrogen, Water Vapor, Liquid Water, and Aqueous Electrolyte Solution Studied by Sum Frequency Generation Spectroscopy. J. Am. Chem. Soc. 2010, 132, 17271-17276.

(33) Kim, J.; Holinga, G. J.; Somorjai, G. A. Curing Induced Structural Reorganization and Enhanced Reactivity of Amino-Terminated Organic Thin Films on Solid Substrates: Observations of Two Types of Chemically and Structurally Unique Amino Groups on the Surface. Langmuir 2011, 27, 5171-5175.

(34) Tong, Y.; Tyrode, E.; Osawa, M.; Yoshida, N.; Watanabe, T.; Nakajima, A.; Ye, S. Preferential Adsorption of Amino-Terminated Silane in a Binary Mixed Self-Assembled Monolayer. Langmuir 2011, 27, 5420-5426. 
(35) Slot, J. W.; Geuze, H. J. A Method to Prepare Isodisperse Colloidal Gold Sols in the Size Range 3-17 nm. Ultramicroscopy 1984, 15, 383-383.

(36) Schneider, C. A.; Rasband, W. S.; Eliceiri, K. W. NIH Image to ImageJ: 25 Years of Image Analysis. Nature Methods 2012, 9, 671-675.

(37) Zhuang, X.; Miranda, P. B.; Kim, D.; Shen, Y. R. Mapping Molecular Orientation and Conformation at Interfaces by Surface Nonlinear Optics. Phys. Rev. B 1999, 59, 12632-12640.

(38) Shen, Y. R. Surfaces Probed by Nonlinear Optics. Surface Science 1994, 299, 551-562.

(39) Guyot-Sionnest, P.; Hunt, J. H.; Shen, Y. R. Sum-Frequency Vibrational Spectroscopy of a Langmuir Film: Study of Molecular Orientation of a Two-Dimensional System. Phys. Rev. Lett. 1987, 59, 1597-1600.

(40) Wei, X.; Hong, S. C.; Lvovsky, A. I.; Held, H.; Shen, Y. R. Evaluation of Surface vs Bulk Contributions in Sum-Frequency Vibrational Spectroscopy Using Reflection and Transmission Geometries. J. Phys. Chem. B 2000, 104, 3349-3354.

(41) Le Rille, A.; Tadjeddine, A. In Situ Visible-Infrared Sum and Difference Frequency Generation at the Electrochemical Interface. Journal of Electroanalytical Chemistry 1999, 467, $238-248$.

(42) Busson, B.; Tadjeddine, A. Non-Uniqueness of Parameters Extracted from Resonant SecondOrder Nonlinear Optical Spectroscopies. J. Phys. Chem. C 2009, 113, 21895-21902.

(43) Tong, Y.; Zhao, Y.; Li, N.; Osawa, M.; Davies, P. B.; Ye, S. Interference Effects in the Sum Frequency Generation Spectra of Thin Organic Films. II: Applications to Different Thin-Film Systems. J. Chem. Phys. 2010, 133, 034705.

(44) Hines, M. A.; Todd, J. A.; Guyot-Sionnest, P. Conformation of Alkanethiols on Au, Ag(111), and Pt(111) Electrodes: A Vibrational Spectroscopy Study. Langmuir 1995, 11, 493-497. 
(45) Pasternack, R. M.; Rivillon Amy, S.; Chabal, Y. J. Attachment of 3(Aminopropyl)triethoxysilane on Silicon Oxide Surfaces: Dependence on Solution Temperature. Langmuir 2008, 24, 12963-12971.

(46) Zhu, M.; Lerum, M. Z.; Chen, W. How To Prepare Reproducible, Homogeneous, and Hydrolytically Stable Aminosilane-Derived Layers on Silica. Langmuir 2012, 28, 416-423.

(47) Palik, E. D. Handbook of Optical Constants of Solids.; Academic, New York, 1985.

(48) Socrates, G. Infrared and Raman Characteristic Group Frequencies : Tables and Chart.; Wiley, 2004.

(49) Weeraman, C.; Yatawara, A. K.; Bordenyuk, A. N.; Benderskii, A. V. Effect of Nanoscale Geometry on Molecular Conformation: Vibrational Sum-Frequency Generation of Alkanethiols on Gold Nanoparticles. J. Am. Chem. Soc. 2006, 128, 14244-14245.

(50) Lu, R.; Gan, W.; Wu, B. H.; Zhang, Z.; Guo, Y.; Wang, H. F. C-H Stretching Vibrations of Methyl, Methylene and Methine Groups at the Vapor/Alcohol $(n=1-8)$ Interfaces. J. Phys. Chem. B 2005, 109, 14118-14129.

(51) Lin-Vien, D.; Colthup, N. B.; Fateley, W. G.; Grasselli, J. G. The Handbook of Infrared and Raman Characteristic Frequencies of Organic Molecules; Academic Press, 1991.

(52) Diesner, M. -O.; Howell, C.; Kurz, V.; Verreault, D.; Koelsch, P. In Vitro Characterization of Surface Properties Through Living Cells. J. Phys. Chem. Lett. 2010, 1, 2339-2342.

(53) Bulard, E.; Guo, Z.; Zheng, W.; Dubost, H.; Fontaine-Aupart, M. -P.; Bellon-Fontaine, M. N.; Herry, J. -M.; Briandet, R.; Bourguignon, B. Non-Invasive Vibrational SFG Spectroscopy Reveals That Bacterial Adhesion Can Alter the Conformation of Grafted "Brush" Chains on SAM. Langmuir 2011, 27, 4928-4935.

(54) Bulard, E.; Fontaine-Aupart, M. -P.; Dubost, H.; Zheng, W.; Bellon-Fontaine, M. -N.; Herry, J. -M.; Bourguignon, B. Competition of Bovine Serum Albumin Adsorption and Bacterial 
Adhesion onto Surface-Grafted ODT: In Situ Study by Vibrational SFG and Fluorescence Confocal Microscopy. Langmuir 2012, 28, 17001-17010.

(55) Backus, E. H. G.; Garcia-Araez, N.; Bonn, M.; Bakker, J. H. On the Role of Fresnel Factors in Sum-Frequency Generation Spectroscopy of Metal-Water and Metal-Oxide-Water Interfaces. J. Phys. Chem. C 2012, 116, 23351-23361.

(56) Liu, Q.; Tang, J.; Zhang, Y.; Martinez, A.; Wang, S.; He, S.; White, T. J.; Smalyukh, I. I. Shape-Dependent Dispersion and Alignment of Nonaggregating Plasmonic Gold Nanoparticles in Lyotropic and Thermotropic Liquid Crystals. Phys. Rev. E 2014, 89, 052505. 


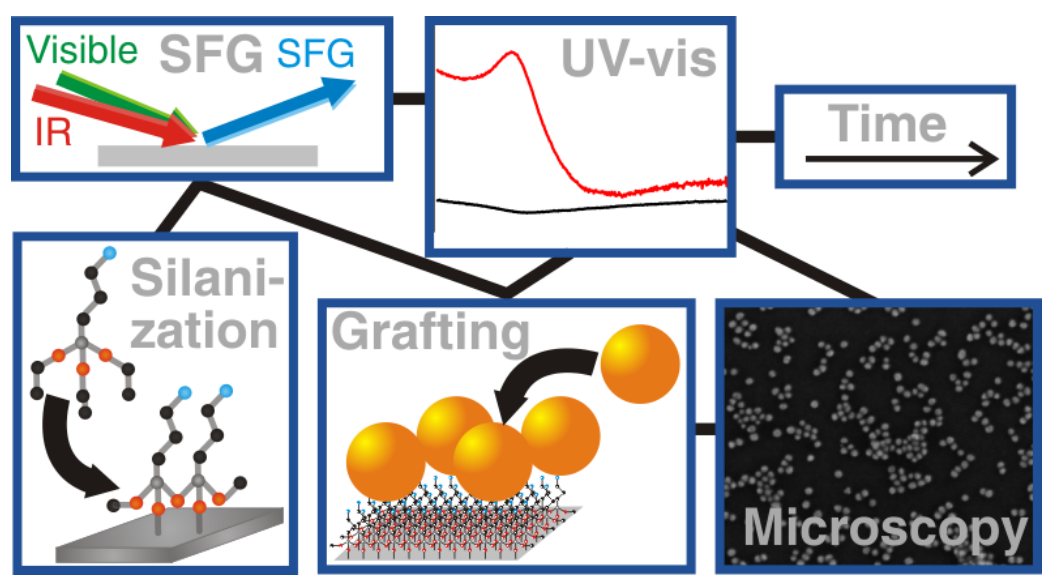

Figure 10: Table of Content

This material is available free of charge via the Internet at http://pubs.acs.org/. 